\title{
Gold in Semiconductor Technology
}

\section{SOME UNCONVENTIONAL METALLURGICAL CONCEPTS}

\author{
G. L. Davis \\ Mullard Limited, Mitcham, Surrey, England \\ In this short general survey of the involvement of gold and its alloys \\ in the complex and widely ranging field of semiconductor devices a \\ metallurgist in the industry has selected those aspects of particular \\ interest from his point of view.
}

To us all gold is known as a precious, handsome and heavy metal which never tarnishes. These attributes scarcely hold charms for a mass production industry; indeed there must be very substantial compensating properties to explain the high and increasing consumption of this metal in an ambiance where minute fractions of a penny added to a factory selling price spread insomnia so relentlessly. Of course, resistance to tarnishing is a consequence of chemical characteristics - complete inertness to liquid and gaseous reagents - which itself engenders obvious merits, and many applications of gold coatings for electronic devices and systems are derived from this property.

Perhaps more interesting, because less familiar, are some of the more esoteric requirements which are met by gold and its alloys. These include high electrical and thermal conductivity, high thermionic work function, relatively low vapour pressure, high diffusion rate into the Group IV elements, "killer" capability for charge carriers in semiconductors, and a convenient range of alloy systems.

The use of gold in semiconductors is more ubiquitous than in the earlier generation of devices based on thermionics which formed the technical and financial springboard for the development and growth of the solid state systems. Even so gold already played a vital part in thermionic devices and before discussing specific semiconductor applications it is perhaps useful to offer a reminder of other involvements of gold deriving essentially from its freedom from oxide films. The most easily recognised applications rested on the external coating of contacting surfaces, while inside the devices, and especially for microwave and power tubes, fabrication still rests heavily on gold-based alloys which provide steps in melting ranges to permit sequential brazing. It is not only that convenient melting points can be obtained, but the alloys, for example with copper, silver, nickel and palladium have easily reduced oxides and can be readily out-gassed. On the whole, too, the phase systems are relatively simple without much intrusion of intermetallics whose presence saps confidence that the mechanical and expansion characteristics will remain stable in life.

A more obscure merit offers advantage. In thermionic devices using oxide cathodes, free barium can evaporate from the cathode and if this deposits on other warm surfaces undesirable origins for electron emission are generated. Gold plating of such surfaces at risk-and this applies especially to control grids-readily takes up barium which then indiffuses and so a surface is maintained of high work function (low emission). For most purposes, a coating of about $0.2 \mu \mathrm{m}$ is adequate and can be applied by electrodeposition or by passing the wire through a bead of molten gold. But undue parsimony can lead to trouble; where barium deposition is heavy, alloys can develop which can melt and migrate. In one extreme case, about 14 per cent barium had been taken up and had given a low melting alloy.

\section{Gold Plated Surfaces}

On semiconductor devices gold plated surfaces are used for the most diverse reasons, and so are laid down under a range of conditions to confer various attributes or to take advantage of several techniques. A consequence is that the plating shop is likely to operate an unusual number of baths ranging from strongly acid right through the $\mathrm{pH}$ range to strongly alkaline. For parts that are sufficiently rugged or awkward to rack, barrel plating is appropriate and neutral baths are then preferred; many finished devices or sub-components may be so treated. Near neutral cyanide baths tend to give the purest and softest gold and are also preferred for troublesome cathode materials which need a strike in unreactive baths; alkaline baths can give higher deposition rates. Baths containing additive can give appropriately "doped" deposits.

Many applications of gold plating are self-evident, conforming to the usual reasons and so will arouse little comment; others have an added interest or 


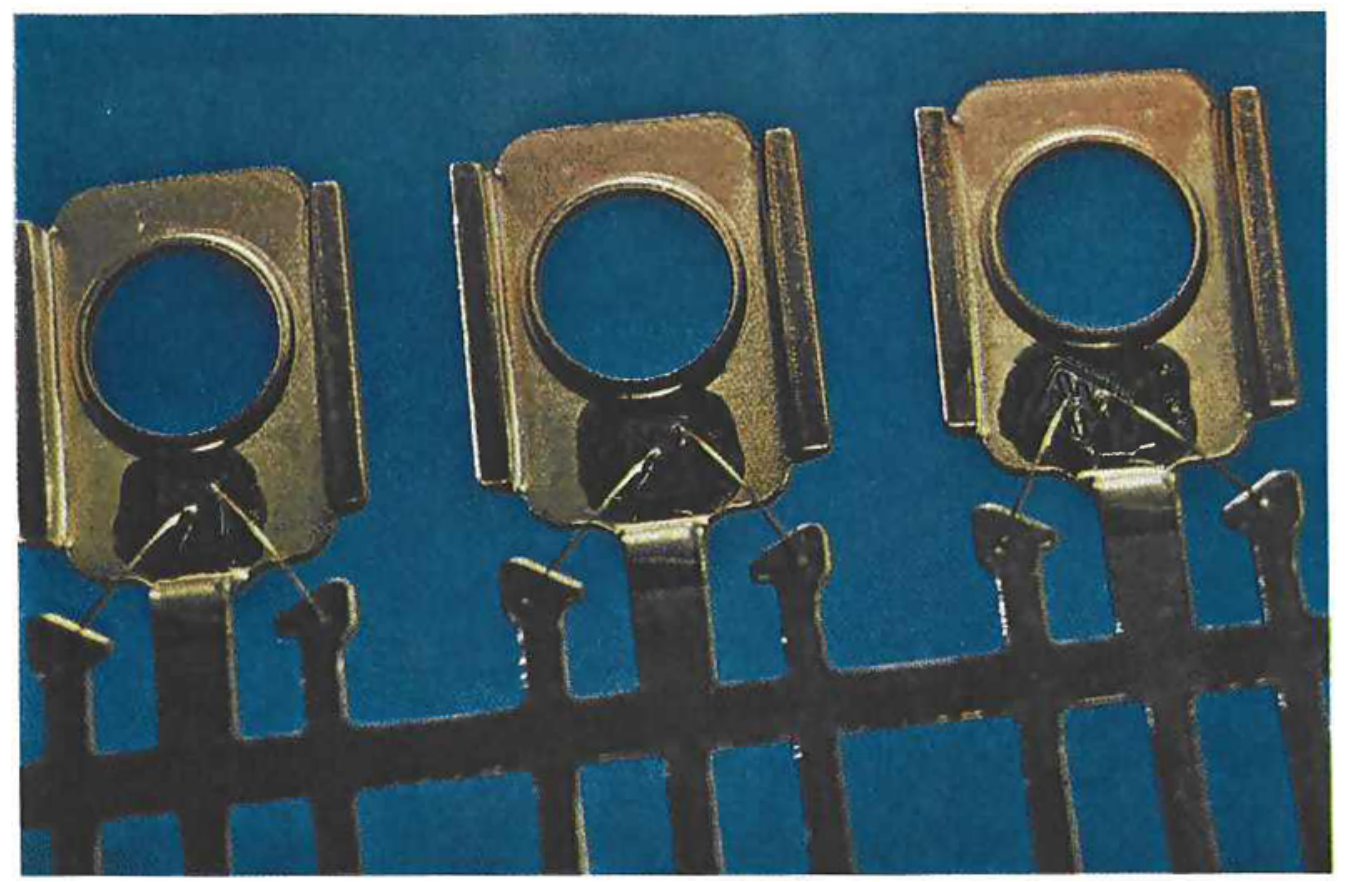

Transistors are normally fabricated on a comb carrying many units, and the devices are tested and encapsulated before being separated. The gold plating of the comb has electrical advantages, assists the bonding and soldering operations and also protects the basis metal during onerous thermal and chemical treatments

unfamiliarity. For instance, extensive use is made of gold as a temporary inert protection against corrosive atmospheres and reagents it is often profitable to coat areas of devices and their assembly components so that they can withstand device processing and then, later, to remove the gold in a recovery plant. In some cases, indeed, selective area coating (predominantly by evaporation) is used as a resist. Where top layers of gold must be photo-mechanically patterned, few substrates will withstand etchants for gold; the gold film together with a supporting sub-layer can sometimes be removed by dissolution of the latter through edge exposures and, perhaps, porosity (photo-resists and aluminium have been so employed).

Most integrated circuits have hitherto used aluminium tracks as conducting paths or pads, and bonding of aluminium and gold wires to such surfaces is generally satisfactory. However, for high reliability and in H.F. devices, there is an increasing

In this close view of a power transistor the semiconductor chip can be more clearly seen soldered to the base metal comb and with its gold lead wires attached tendency to replace these tracks with gold evaporated and then plated up for certain cases, and then it may assist bonding to deposit the harder golds, although it is usually preferred to avoid the alloying additions as a hardening method. One factor improving reliability is that gold will not sustain electro-migration, a phenomenon which is a problem with aluminium.

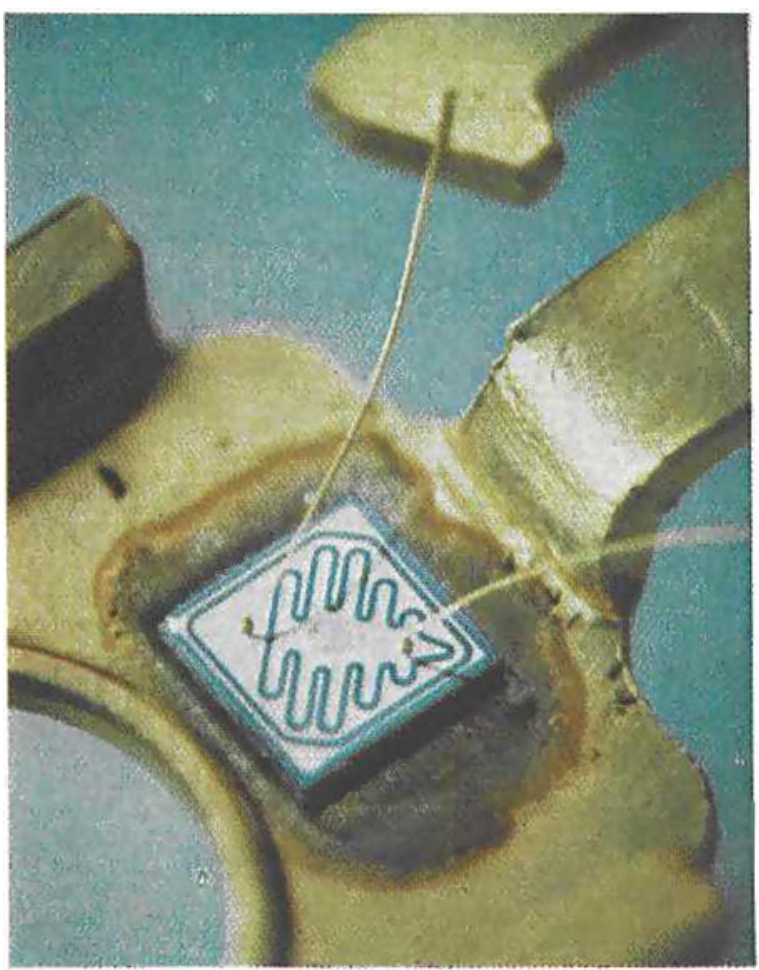




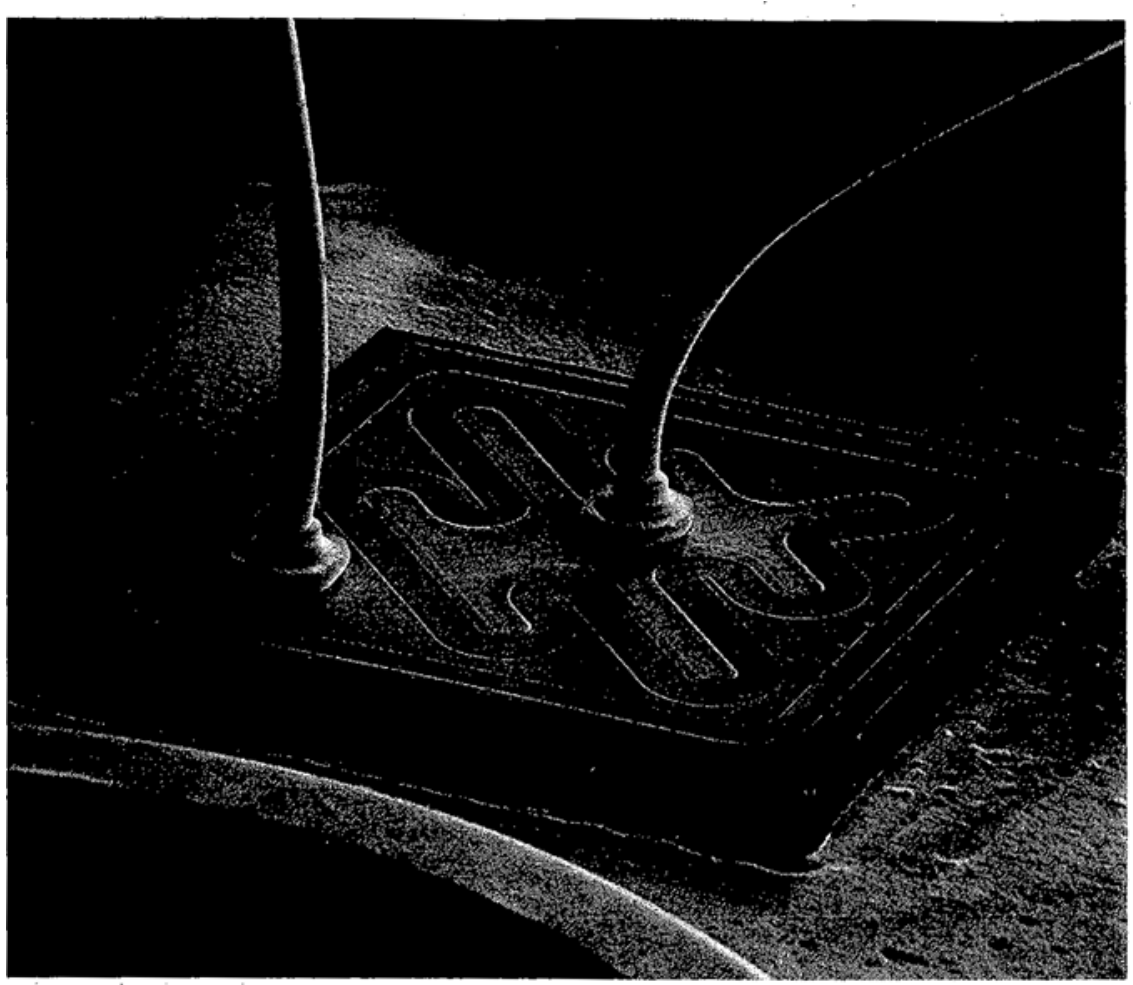

A scanning electron microscope view of a power transistor which is brazed to a heavily gold-plated copper support to act as a heat sink. The collector, dissipating most heat, is the back connection and the two nail-head thermo compression bonded leads are for the base and emitter zones, the latter lying within the "meander" pattern which affords a long junction length. Ohmic contact to the elec. trodes is developed, as the semiconductor is coated with a film of aluminium beneath the bonds. The dark tracks are deeply coloured gilicon oxide films

$\times 100$

Bonding of chips (integrated circuits or individual devices) on to headers can involve "large" area joining of the back side of the chip. For the less demanding devices soft solders are suitable, and then preplating of the chip and surfacing of the header with gold is

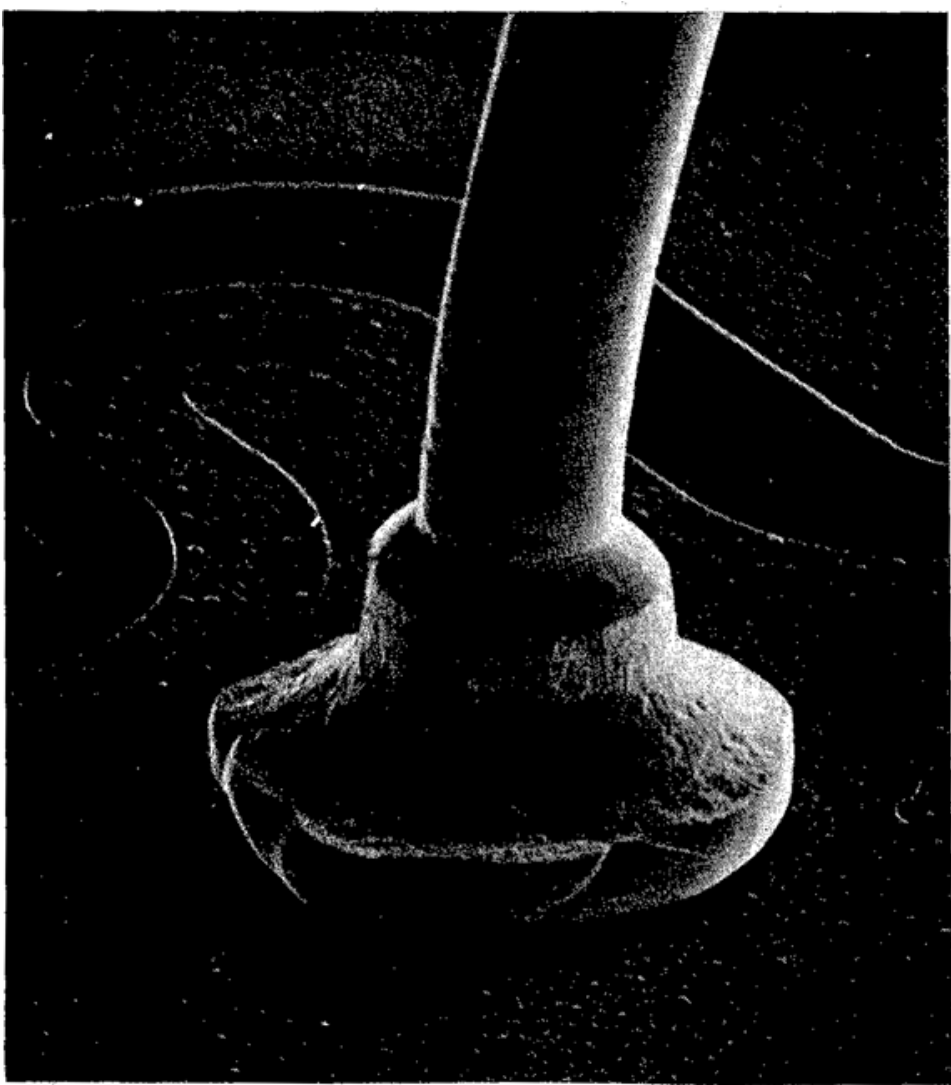

commonly practised, keeping thicknesses down to levels appropriate to avoid embrittlement from tingold intermetallics. More rigorous demands are met with a bond by pre-forming the convenient goldsilicon eutectic (melting point $370^{\circ} \mathrm{C}$ ) and this gives a strong bond with low thermal and electrical resistance and excellent performance on thermal cycling, despite the dilatometric mismatch between the low expansion silicon and the header (often copper). To improve the behaviour further, it is common to interpose a low expansion plate of nickel-iron alloy or molybdenum. These are prefabricated from strip coated with say $4 \mu \mathrm{m}$ of gold on the face to take the silicon and perhaps 0.2 to $0.4 \mu \mathrm{m}$ on the face to be soft soldered. Other foils may have much greater thicknesses of gold (for example up to $20 \mu \mathrm{m}$ ) on one side and solder layers deposited on the other.

Hybrid circuit modules fabricated by the thin film route may use titanium on the ceramic or glass,

Pure gold wire, $37 \mu \mathrm{m}$ in diameter, has been fused, by a flame or a spark, to give a ballend of ahout $150 \mu \mathrm{m}$ and this has been used to make a thermocompression bond to the aluminium surfaced emitter contact of this power transistor 
Gold-bonded diodes rely on local melting to form a eutectic alloy between the semiconductor chip (doped in one sense) and gold wire carrying a small content of dopant (of opposite sense). The fusion is generated after completing the assembly of the device in its glass envelope. Part of the envelope wall has been removed to illustrate the structure in this scanning electron microscope picture $\quad \times 120$

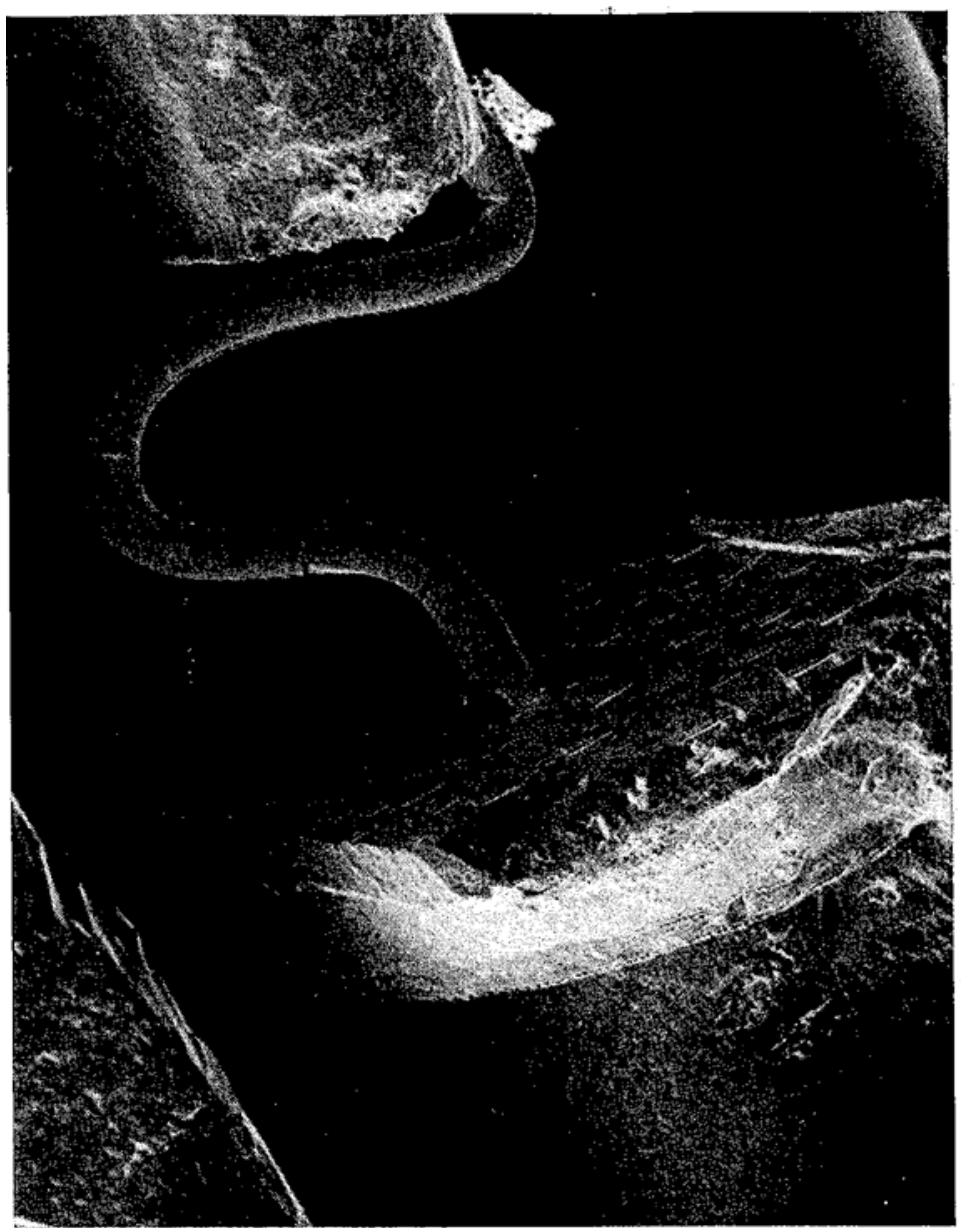

coated with gold for conductors and etched locally after photo-mechanical processing to leave titanium resistors.

\section{Gold and Silicon}

When gold-coated silicon slices are heated, deliberately or as part of the slice processing, indiffusion is comparatively rapid. This leads to phenomena both useful and inconvenient, and they have been the subjects of many investigations.

First, gold acts as a p-type impurity so that this may require pre-doping the metal to ensure ohmic contacts. Significant changes in electrical characteristics are conferred at very low concentrations and at solubility levels minute by customary metallurgical standards, for example at $10^{15}$ to $10^{18}$ atoms $/ \mathrm{cm}^{3}$, and are then sensitive to the presence of third elements such as boron and phosphorus. Since the latter (and other elements) are located in zones adjacent to junctions, there can be a segregation of gold which modifies the normal error-function edge of the diffusion and leads to concentration steps with factors of perhaps an order.

In cases where gold diffusion must be prevented, a barrier is interposed of evaporated metal such as nickel (or better cobalt) on to copper surfaces: Between silicon and gold, evaporated titanium is moderately satisfactory as it adheres well to silicon (even if oxidised) and to n- and heavily p-type gives ohmic contact. But, while still in the evaporator, a follow-up metal can be deposited such as platinum, which offers a better barrier and whose diffusion into the gold is slow and into silicon prevented by the titanium. Devices with gold beam leads can be made by gold plating up the platinum coating evaporated on to the titanium film. As both titanium and platinum react too rapidly with tungsten at the temperatures required, these metals are best evaporated by electron-beam bombardment of a source.

\section{Gold as "Killer"}

In-diffused gold acts in a second way. In semiconductor devices the signal is carried by extra injected minority carriers (electrons or holes according to type) and they diffuse down the concentration gradient set up in the equilibrium ratio of holes and electron previously obtaining. To re-establish equilibrium, recombination must occur and this can be too slow to prevent degrading of high frequency pulse shapes. Very dilute dispersions of gold set up 


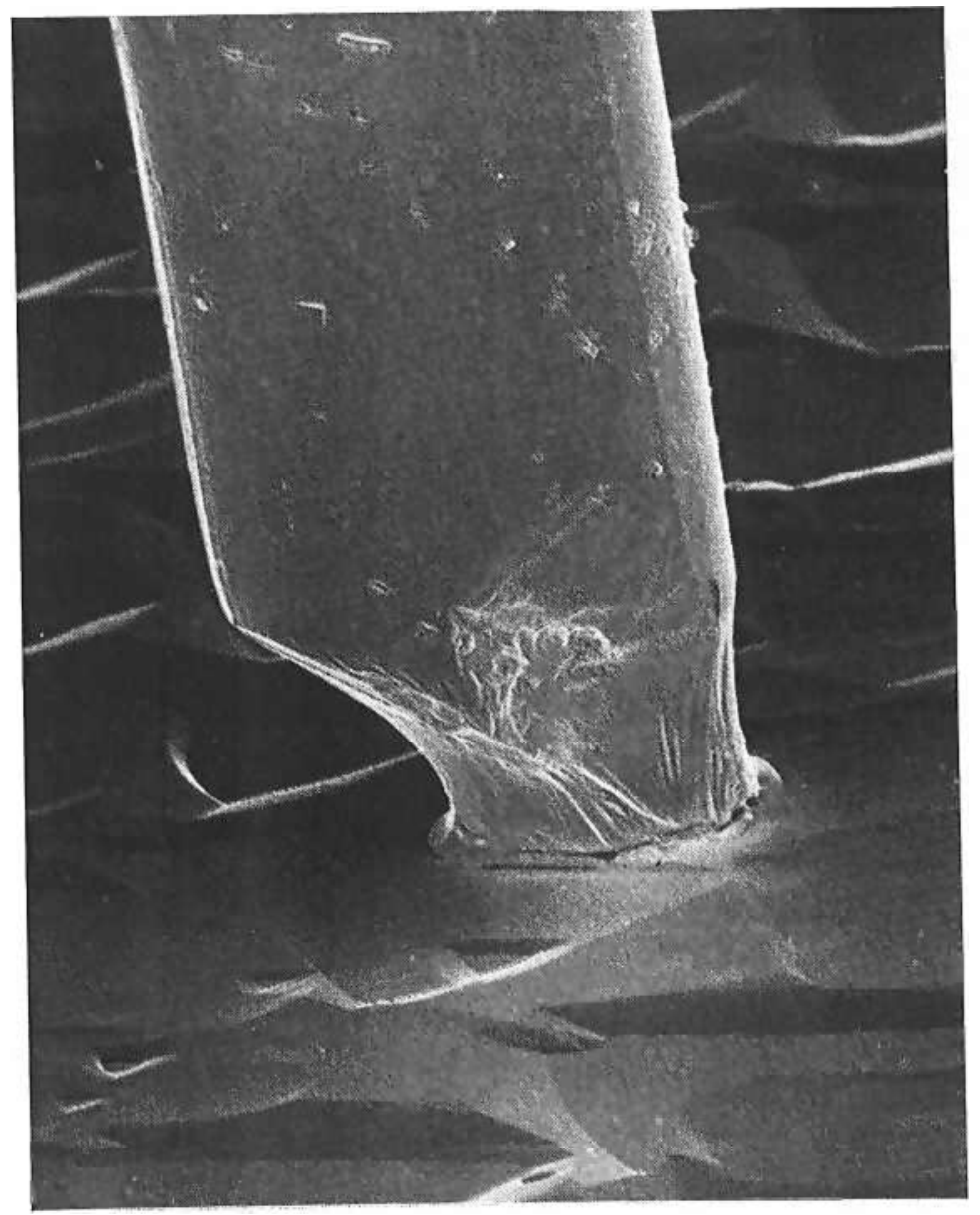

This scanning electron microscope illustration shows the detail of the join between the doped gold and the surface of the germanium chip. When the wire is pressed on to the chip and a current pulse is passed the zone of contact fuses to produce a low melting eutectic which solidifies to give an epitaxial layer of now doped germanium on the original crystal, so forming the required p-n junction $\times 1200$

energy levels within the forbidden band acting as "fast traps" within which recombination is facilitated and so the effective lifetime of carriers is decreased.

Advantage can be taken of this behaviour, by evaporating gold (from a bead on a tungsten filament) on to the back of a slice, typically to a thickness of a few hundred $\AA$ and in-diffusing at over $1000^{\circ} \mathrm{C}$, e.g. for some logic integrated circuits and fast switching transistors for high frequency devices.

\section{The Gold-Silicon and}

\section{Gold-Germanium Eutectics}

As mentioned above, the gold-silicon phase diagram shows a eutectic of composition approximately 3 per cent silicon melting at $370^{\circ} \mathrm{C}$ and with

This section of a gold-bonded diode shows how some of the semieonductor has been removed by the formation of the low melting eutectic when the tip of the wire was electrically melted. Germanium crystals can be seen throughout the resolidified fused zone, but a very thin epitaxial deposit of low-doped germanium on the cavity in the chip provides a p-n junction

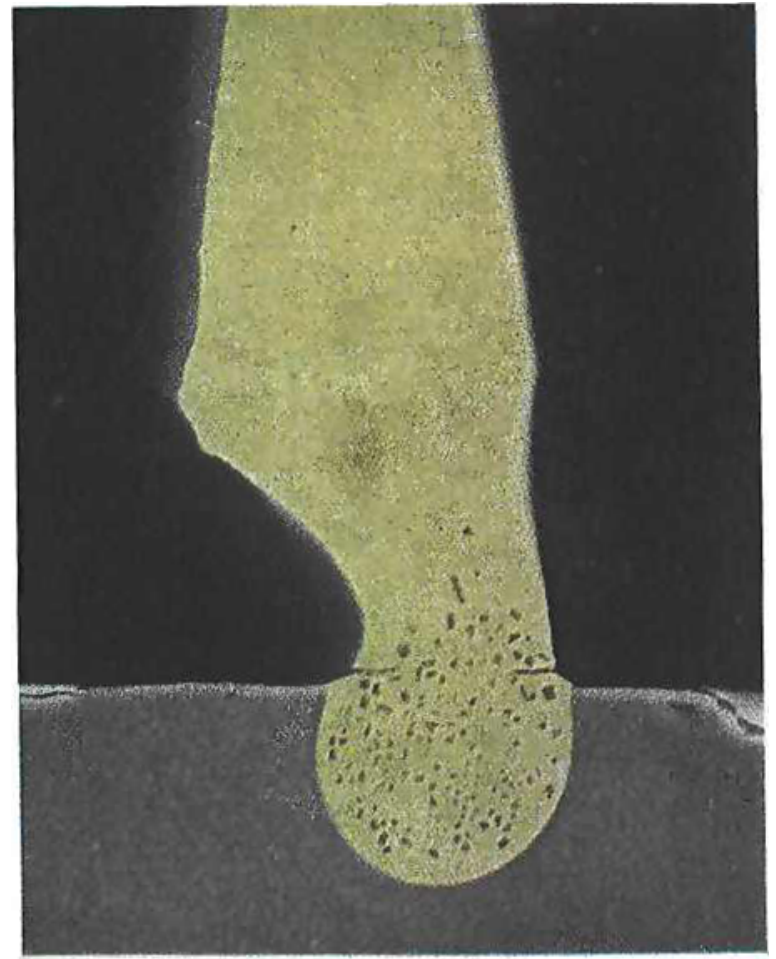


The gold-silicon equilibrium diagram shows that the terminal solubility of silicon is low so that when gold is melted on to a silicon semiconductor chip almost pure components separate out on cooling. The gold deposited is therefore of good conductivity, and use can be made of the depositing semiconductor if it grows epitaxially on to the parent wafer to generate p-n junctions. The convenience of the low melting eutectic facilitates gold brazing of devices to support headers and gives strong, sound joints of low thermal resistance and with ohmic contact. The gold-germanium equilibrium diagram is very similar in form in the location and temperature of the eutectic. The terminal solubility of germanium is again low

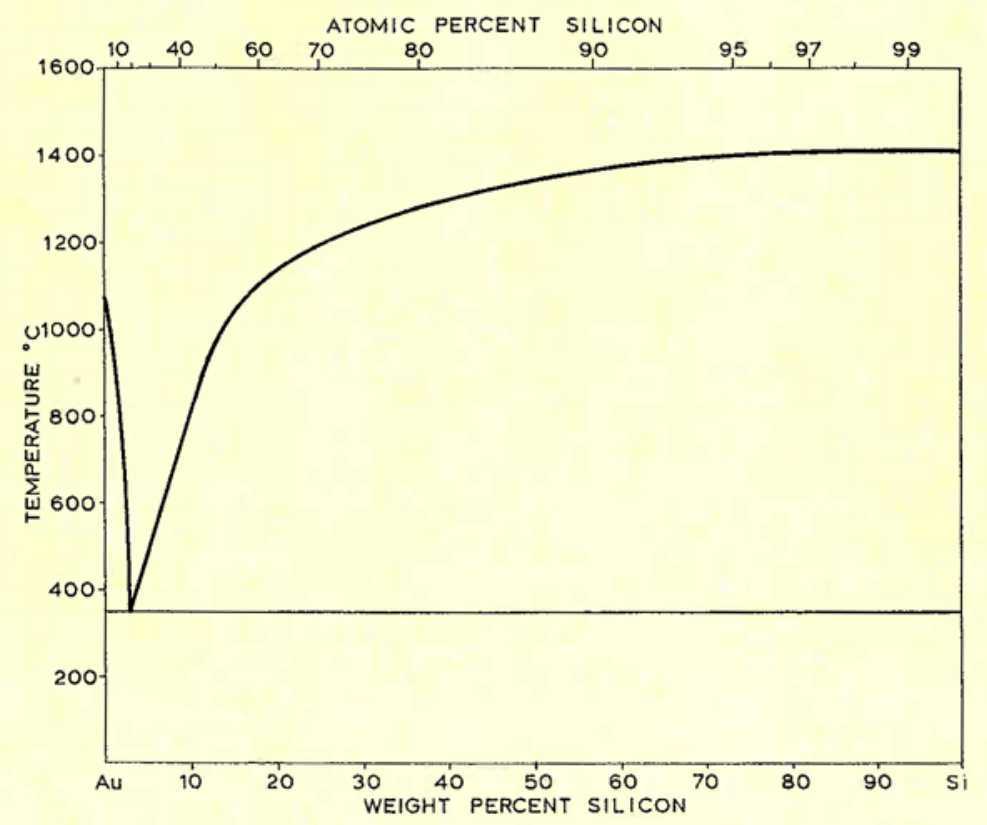

extremely low terminal mutual solubilities. This surprisingly low melting alloy offers an extremely convenient route for bonding silicon to substrate metals and, as the solubility of silicon in gold is so low, a high thermal conductivity is maintained which spreads local heating of the device. This, coupled with fortunate mechanical characteristics of the alloy, gives a bond which is perhaps the most trouble-free of any under thermal cycling, despite the expansion mismatch and the relatively large temperature drop from the freezing point to operating temperatures. A similar situation arises with the gold-germanium system.

A more subtle use can be made of such alloying for so-called gold-bonded diodes. In these, a pointed gold wire is pressed on to a germanium slice and given an energy pulse sufficient to cause melting at the contact point. Germanium is dissolved in the gold and re-precipitated on cooling, at least partially epitaxially on the unmelted slice. If the gold wire used carries a small concentration of a suitable third element, the re-precipitated germanium zone can be of such type as to give a sharp p-n junction. Typically, gallium-doped gold can be used on to n-type germanium.

\section{Gold Wire Bonding}

Affixing gold wire flying leads on to microscopically small devices has bred a revolution in "cold welded" bonds. Initially effected manually, these are now largely automated by the development of high precision machines and raw materials of controlled and consistent quality. Gold wires are often used for thermo-compression bonding and especially "nailhead" bonding. In both, the wires are heavily deformed under pressure on to the contact. surface, which may be gold but is more commonly aluminium. The elegant nail-head bonder feeds fine wires (down to $18 \mu \mathrm{m}$ but also up to $500 \mu \mathrm{m}$ ) through a capillary and by fusion of the protruding end leaves a spherical termination, which is then pressed on to the device heated to around $300^{\circ} \mathrm{C}$. Fusion may be by a tiny. hydrogen flame or by Joule heating from a capacitor discharge. Similarly good bonds, but with greater deformation, can be made at lower-indeed at roomtemperature, if ultrasonic rubbing is incorporated. This is preferred for more thermally sensitive devices if bonds do not have to be too close together.

As made, both types of bond are excellent, but care is needed that the somewhat necked and fully annealed wire above the joint is not unduly stressed or repeatedly flexed, and this can happen on poorly encapsulated devices, thermally cycled. It is cläimed that spark balling tends to reduce grain growth in these sensitive zones and hence to enhance strength.

\section{The Gold-Aluminium Interaction}

At this point it is opportune to introduce the dreaded "purple plague", with the hope of presenting the phenomenon in reasonable perspective.

The gold-aluminium system is complex, with several intermetallic compounds, and, as is not uncommon, these tend to be rather brittle. When these develop as discrete bands, as is inevitable in a diffusion couple at elevated temperatures, the interfaces can be sources of low shear strength and 


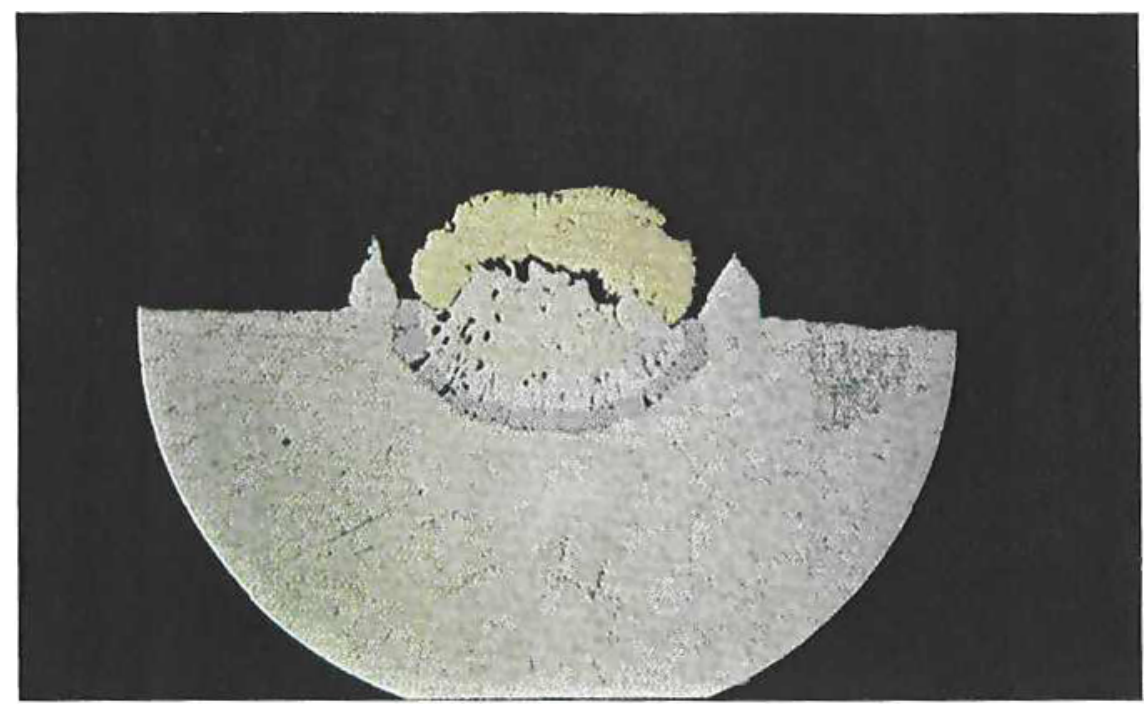

Some intermetallic compounds of gold and aluminium are highly coloured so that their presence in a diffusion system can be readily recognised. This section of a diffusion zone between a gold wire and aluminium-coated silicon shows a band of the coloured compound $\mathrm{AuAl}_{2}$ popularly known as "purple plague"

these effects perhaps can be aggravated by the generation of cavities due to disparate diffusion rates (the Kirkendall effect). Some of these phases are intrinsically coloured and produce intense bands, mainly shades of purple $\mathrm{AuAl}_{2}$, but even black, on superficial examination; less so in sections. Pretty demonstration pieces can be made by heating evaporated sandwiches of alternate gold and aluminium films in a few hours at around $200^{\circ} \mathrm{C}$.

Since the rates of formation of these phases follow the usual exponential laws with activation energies in the region of $15 \mathrm{kcal} / \mathrm{g}$-atom on bulk samples, it is only when devices operate at higher temperatures that degradation is serious, when practical experience with thin films tends to give results indicative of diffusion more rapid than predicted. $\mathrm{Up}$ to $80^{\circ} \mathrm{C}$, a life of about $10^{5} \mathrm{~h}$ may be expected, but above say $150^{\circ} \mathrm{C}$ the effects can be unacceptable. Some evidence suggests that development of the purple phases, with which failure is mainly associated, is somewhat accelerated by the presence of silicon; compounds containing some 6 per cent silicon have been observed. Degradation can also be aggravated by the retention of reagents or encapsulant compounds, it is supposed mainly by enhancing fissuring due to stress corrosion.

This apparent disastrous development of plague is perhaps now viewed more tolerantly; if the extent is slight, indeed, a not uncommon viewpoint is that its manifestation illustrates that bonding has been effective. Of course, there remains the possibility of interposing barriers between the two metals and among others molybdenum or tungsten have been suggested. Alternatively, the device contact may use gold and avoid the problem.

This account of the usage of gold in the semiconductor industry evidently cannot pretend to be exhaustive, but it is hoped that the more uriconventional metallurgical concepts raised in this survey will promote further interest.

\section{Acknowledgments}

I am indebted to many colleagues having close and expert contact with the subject, with whose generous assistance I have prepared this account. In particular I wish to acknowledge help from Mr I. Baldwin, Dr R. Jones and $\mathrm{Mr}$ C. Oliver of our associated company Mullards Southampton and to thank the Directors of Mullards for permission to publish this paper.

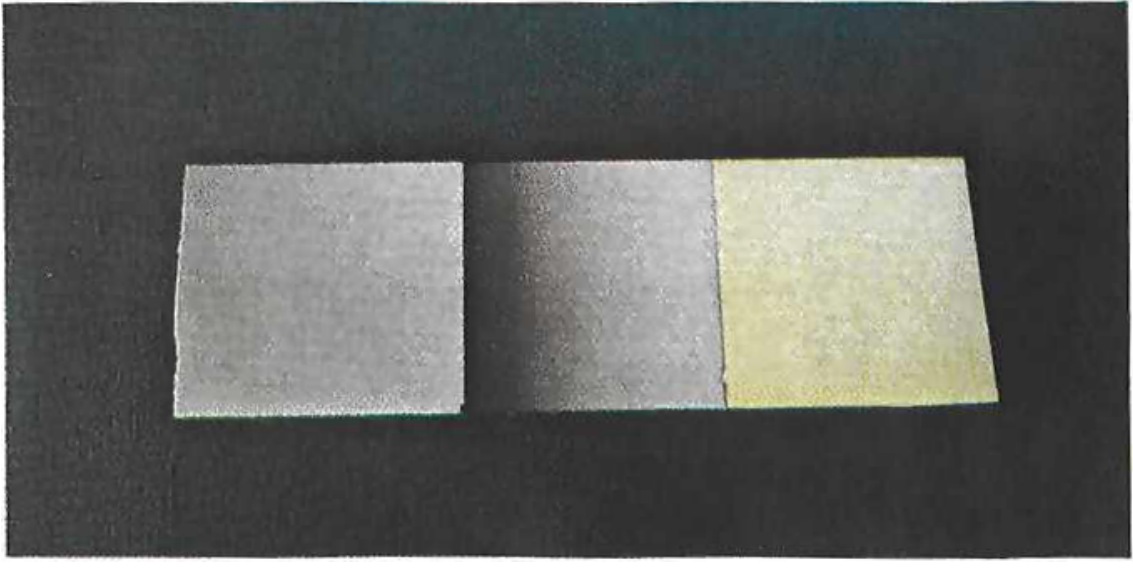

This illustration of the gold. aluminium purple compound was made by heating at $200^{\circ} \mathrm{C}$ for a few hours a microscope slide on which was evaporated sandwiches of aluminium, gold and aluminium. The two component metals are on either side of the band showing the coloured compound 